\title{
Photon-Spin Control of Quantum-Dot Emission with Nanoantennas
}

\author{
Sergey S. Kruk, Manuel Decker, Isabelle Staude, Stefan Schlecht, Michael Greppmair, \\ Dragomir N. Neshev, and Yuri S. Kivshar \\ Nonlinear Physics Centre, RSPE, The Australian National University, Canberra, ACT 0200, Australia \\ Sergey.Kruk@anu.edu.au
}

\begin{abstract}
We experimentally demonstrate spin-polarized light emission from quantum dots coupled to a single-element metamaterial nanoparticle with resonant multipolar moments. We observe spin-momentum locking resulting in photons of opposite spin emitted in opposite directions.
\end{abstract}

\section{INTRODUCTION}

Nanoantennas improve dramatically the sencitivity of fluorescent detection from single emitters, such as molequles and quantum-dots. Antennas enable the efficient transfer of the fluorophore emission from the near- to the far-field and vice versa. More importantly nanoantennas enable large enhancement of the spontaneous emission rates [1], as well as an unprecedented control of the directionality of light emission [2]. Even more, optical nanoantennas allow for control of the spin polarization state of fluorophore emission, which can be of particular interest when transitions from different spin levels have to be examined.

The conventional way for the generation of circularly polarized radiation is to employ helical antennas, however the spin of emitted photons is fixed by the antenna helicity. Other concepts are based on phase-retardation between multiple dipolar antennas, however these are highly sensitive to the exact positioning and orientation of the emitter. Overall, there is no experimental demonstration of chiral light emission that allows for reliably controlling the spin of photons (ellipticity and polarity of polarization) emitted from nanoscale light sources coupled to nanoantennas.

Here, we propose and demonstrate a novel concept of manipulation of the polarization state of light emission in a system of a semiconductor quantum-dots (QDs) coupled to a single-element multipolar nanoantennas. In particular, we show that the coupling of QDs to a plasmonic nanoantenna, which is characterized by a superposition of several resonant multipolar contributions (e.g. electric/magnetic dipole moments, quadrupole moments etc.) with a fixed phase difference can lead to emission of chiral light of opposite handedness from the coupled QDnanoantenna system. Furthermore, the spin of the emitted photons is insensitive to precise positioning of the emitter relative to the antenna, which is of practical importance. More specifically, the superposition of the nanoantenna's multipole radiation patterns in the far-field determines the ellipticity and handedness of light emitted from the QDs in different directions, hence, allowing the selection of photons with specific polarization properties in the Fourierspace. Our concept opens a new route to nanoscale light sources of spin-polarized photons as well as for simple recognition of emission from chiral emitters of different handedness.

\section{RESUlts AND Discussions}

Our novel approach for chiral light generation is based on a multipolar nanoantenna driven in the near-field by an electric dipole emitter, e.g. a QD. The far-field emission pattern of such a coupled system is dominated by the emission from the nanoantenna's electric and magnetic multipoles, which are significantly larger than the dipole moment of the QD. At resonance, the far-field emission is hence a superposition of the main antenna multipolar contributions: electric dipole $(\vec{p})$, magnetic dipole $(\vec{m})$, and electric quadrupole $(\widehat{Q})$,

$$
\vec{E}(r)=\left(k_{0}^{2} e^{i k_{0} r} / 4 \pi \varepsilon_{0} r\right)\left([\vec{n} \times[\vec{p} \times \vec{n}]]+\sqrt{\varepsilon_{0} \varepsilon \mu_{0}}[\vec{m} \times \vec{n}]+\left(i k_{0} / 6\right)[\vec{n} \times[\vec{n} \times \widehat{Q} \vec{n}]]+\ldots\right),
$$

where $\vec{n}$ is the unit vector in the direction of emission, $r$ is the coordinate and $k_{0}$ is the photon wavevector. We assume that the electric dipole $\vec{p}$ is oriented along $x$-axis, the magnetic dipole $\vec{m}$ points in $z$-direction, and the 

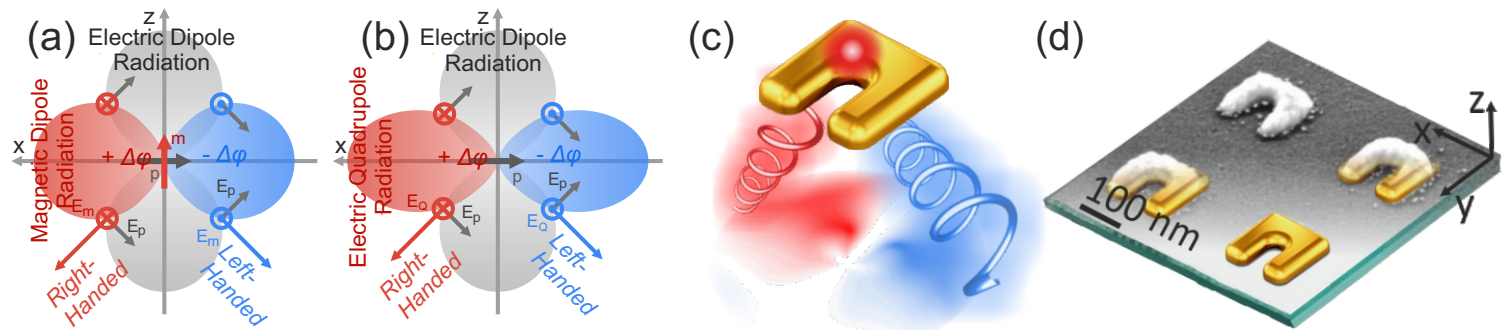

Fig. 1: Electric field cross section, superposition from (a) orthogonal electric and magnetic dipoles; (b) electric dipole and square quadruple. The field components from two multipoles are orthogonal and have $\pi / 2$ phase difference. (c) Schematic of the QD-SRR coupled system. (d) Scanning electron micrograph of the SRR array.

quadrupole tensor has two non-zero components $Q_{x y}=Q_{y x}$. Eq. (1) shows that the electric field contributions from the electric and magnetic dipoles [Fig. 1(a)] or the electric dipole and quadrupole [first and third term in Eq. (1) - Fig. 1(b)], for given orientations are perpendicular to each other. For a $\pi / 2$ phase difference between them the electric field is elliptically polarized with the ellipticity depending on the relative amplitudes of the multipoles. Importantly, in a single-element nanoantenna the relative phases of the multipolar moments are fixed with respect to each other as they correspond to a unified distribution of currents and charges in the antenna. Therefore by altering the multipole components of the nanoantenna it is possible to control the polarization of the emitted light.

To prove this concept experimentally, we employ split-ring-resonators (SRRs) as multipolar nanoantennas and couple them to semiconductor QDs [see Fig. 1(c)] emitting at around $800 \mathrm{~nm}$ wavelength. We use electron-beam lithography to fabricate arrays of gold SRRs on a glass substrate [Fig. 1(d)] and cover them with a thin $\mathrm{MgF}_{2}$ spacer layer and a uniform layer of core-shell CdSeTe QDs on top.

The SRRs exhibit a number of resonances in the near-infrared spectral range [3, 4]. We choose the so-called second order resonance, which is dominated by a superposition of an electric dipole and an electric quadrupole [4]. These two multipoles fulfill the aforementioned requirements of perpendicularity and phase difference [Fig. 1(b)]. We chose the design of the SRR such that this resonance spectrally overlaps with the emission of QDs and can therefore be employed for generation of spin-polarized photons. For vertical $y$-polarization, there is a single broad resonance at $\lambda_{1} \approx 815 \mathrm{~nm}$, while for horizontal polarization there are two resonances at $\lambda_{2}=760 \mathrm{~nm}$ and $\lambda_{3}=620 \mathrm{~nm}$ wavelength [see Fig. 2]. The resonance $\lambda_{1}$ is dominated by a single strong electric dipole moment (along $y$-direction), while resonance at $\lambda_{2}$ is characterized by a superposition of an electric dipole (along $x$-direction), an electric quadrupole with two non-zero components $Q_{x y}=Q_{y x}$, and a weak magnetic dipole moment (along $z$ direction). The scattering contribution of the SRR at the QD emission wavelength is therefore dominated by electric dipole and quadrupole contributions. Since the fields from the dipole and the quadrupole components are perpendicular to each other and $\pi / 2$ phase-shifted [Fig. 1(b)], the SRR nanoantenna can generate spin-polarized photons by coupling to QDs. We note that the SRR fundamental resonance [3,4] is at $1400 \mathrm{~nm}$ and is dominated by an electric and magnetic dipole moment [similar to Fig. 1(a)]. This could also be used to manipulate the spin of the light emission, however it is less efficient.

To investigate the emission properties of the QDs coupled to the SRRs without affecting the excitation rate, we
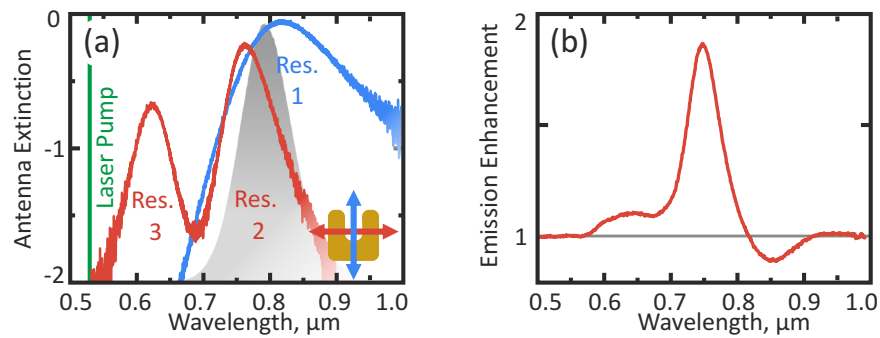

Fig. 2: (a) Extinction spectra [ $\ln (1-\mathrm{T})]$ of the SRRs array for horizontal (red) and vertical (blue) polarizations (see inset). The spectra are shaped by three resonances at wavelengths $\lambda_{1} \approx 815 \mathrm{~nm}, \lambda_{2} \approx 760 \mathrm{~nm}$ and $\lambda_{3} \approx 620 \mathrm{~nm}$. The gray-shaded region shows the emission spectrum of the QDs, which is in overlap with the resonances $1 \& 2$. (b) Photoluminescence (PL) of the coupled system of QDs with SRRs (red line) referenced to the PL of the uncoupled QDs on the glass substrate. 
pump them optically at $532 \mathrm{~nm}$, i.e. well below the SRR resonances. We collect the photo-luminescence (PL) of the QDs from the substrate side and resolve the PL in Fourier-space ( $k$-space). As a reference we measure the PL of the QDs on a plane glass substrate. We measure the ellipticity of the PL for every point of the experimentally accessible part of the $k$-space [Fig. 3(a)] via the Stokes vector formalism. Along the symmetry plane of the SRR $\left(k_{x}=0\right)$ the emission is linearly polarized, while for $k_{x} \neq 0$ the emission becomes elliptic with ellipticity reaching 0.5 . The polarization pattern is mirror symmetric to the $k_{x}=0$ plane and for $k_{x}<0$ the emission is right-handed elliptically polarized, while for $k_{x}>0$ it is left-handed elliptically polarized. We also find that the polarization inclination qualitatively follows this symmetric behavior, exhibiting opposite inclinations in opposite semi-planes $\pm k_{x}$. We also observe a non-negligible amount of depolarizion in the experiment that can be attributed to the incoherent superposition of resonance 1 and resonance 2 of the SRRs in the array as well as to the contribution of the uncoupled QDs. This depolarization, however, vanishes for a single SRR nanoantenna coupled to a single QD.
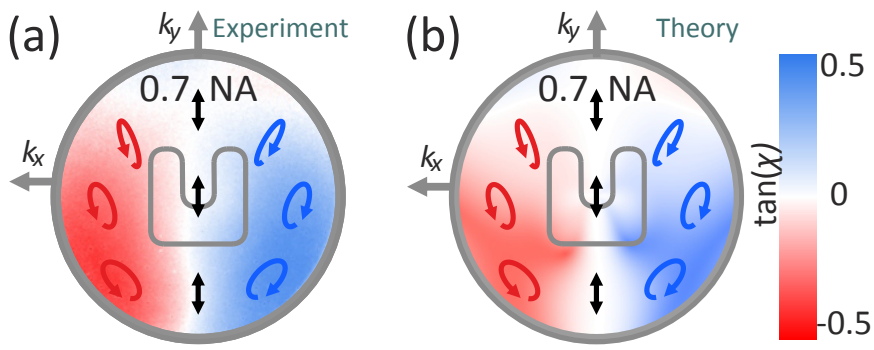

Fig. 3: (a) Experimentally measured and (b) theoretically calculated ellipticity of emission of the coupled SRR-QD system. Blue/red color corresponds to left/right-handed elliptical polarization, respectively; white color - to linear polarization. The insets in the middle show the orientation of the SRR.

Furthermore, we perform numerical calculations, where we place a single small dipole source at a height of $14 \mathrm{~nm}$ over the center of a single SRR with the dimensions corresponding to the fabricated ones on a substrate with a refractive index of $n=1.41$, covered with $12 \mathrm{~nm}$-thick $\mathrm{MgF}_{2}$ layer with refractive index $\mathrm{n}=1.38$. We then and calculate the 3D electromagnetic far-field distribution of the coupled system. To match our experiments, we average over all dipole orientations (dipole pointing in $x-, y$-, and $z$-direction) and calculate the average value of ellipticity. These results in Figure 3(b) show the complementary numerical calculations of the PL polarization state, which well reproduce our experiments. Importantly, our calculations also predict that the emission can be purely circularly polarized in specific directions for an optimized system.

\section{CONCLUSION}

We have demonstrated that by coupling a QD to a multipolar SRR nanoantenna we can create spin-polarized light emission from a subwavelength light source. We make use of the phase-locked superposition of several multipole contributions of the SRR at resonance to obtain direction-dependent spin-polarized PL of QD. This effect is inherently robust against the exact position of the emitter, being strong even for emission from random distribution of QDs on a SRR array. Our novel concept is also applicable to other types of nanoantennas that support multipolar resonances and enables new opportunities for generation and detection of spin-polarized light.

\section{ACKNOWLEDGEMENT}

We acknowledge the support from the Australian National Fabrication Facility and the Australian Research Council. The authors thank A. Miroshnichenko, A. Evlyukhin, C. Rockstuhl, A. Chipoulin, R. Schiek and A. F. Koenderink for the useful discussions.

\section{REFERENCES}

[1] P. Anger et al., "Enhancement and quenching of single-molecule fluorescence," Phys. Rev. Lett. 96, 113002 (2006).

[2] A. G. Curto et al., "Unidirectional emission of a quantum dot coupled to a nanoantenna," Science 329, 930-933 (2010).

[3] S. Linden et al., "Magnetic response of metamaterials at 100 terahertz," Science 306, 1351-1353 (2004).

[4] M. Decker et al., "Dual-channel spontaneous emission of quantum dots in magnetic metamaterials," Nat. Commun. 4, 2949 (2013). 